ISSN 0258-7122

Bangladesh J. Agril. Res. 39(1): 93-103, March 2014

\title{
EFFECTIVENESS OF INDIGENOUS PLANT POWDERS AS GRAIN PROTECTANT AGAINST Callosobruchus chinensis (L.) IN STORED CHICKPEA (Cicer arietinum)
}

\author{
M. A. $\operatorname{HOSSAIN}^{1}$, M. A. A. BACHCHU ${ }^{2}$ \\ K. S. AHMED ${ }^{3}$ AND M. A. HAQUE ${ }^{4}$
}

\begin{abstract}
The effectiveness of 17 indigenous plant powders as grain protectant were assessed against Callosobruchus chinensis (L.). The results indicated that among all the tested plant materials, tobacco leaf powder (TLP) had promising effects on inhibiting oviposition and reducing adult emergence, seed infestation, and weight loss by $C$. chinensis. Tobacco leaf powder offered complete protection of chickpea seeds applied at $20.0 \mathrm{~g} / \mathrm{kg}$ seeds. Its lower doses exhibited efficacy in dose dependant manner. The lowest number of eggs (24.60), egg bearing seeds (23.40), adult emergence (23.20), seed infestation (8.28\%), and weight loss $(0.50 \%)$ were obtained from the TLP treated at $10.0 \mathrm{~g} / \mathrm{kg}$ seeds, while the highest of these parameters were in untreated control. In the ovicidal test, TLP showed $100 \%$ inhibition at $20.0 \mathrm{~g} / \mathrm{kg}$ seeds over control. The lowest number of adults (37.20) were emerged when larvae bearing seeds were treated with TLP at $20.0 \mathrm{~g} / \mathrm{kg}$ seeds along with $59.39 \%$ retardation over the control and had no adverse effect on seed germination up to 3 months.
\end{abstract}

Keywords: Plant powders, chickpea seeds, protectant, Callosobruchus chinensis.

\section{Introduction}

Pulses play a pivotal role in the diet of common people of third world country including Bangladesh. These are also called "poor man's meat" since they are rich source of protein (20-40\%) and are fairly good sources of thiamin, niacin, calcium, and iron for the under privileged people who cannot afford animal proteins (Sharma, 1984; Bhalla et al., 2008). Different types of pulses are grown throughout the winter season in Bangladesh and produce a total 2,05,000 $\mathrm{m}$ t of pulses. Among the pulses, chickpea alone occupied $4.06 \%$ area of pulse cultivation and contributed $7000 \mathrm{~m}$ t of annual production (BBS, 2008). But one of the major limitations for increasing pulses production is losses of seed viability and damage of grains from insect pest infestation in storage. Pulses in developing countries suffer high qualitative and quantitative losses from the attack of pulse beetle, Callosobruchus chinensis L., a major pest of pulses in storage (Ahmed et al., 2003; Aslam, 2004). They cause damage to pulses both in the field and storage, but infestation is more crucial in stored condition (Rahman

${ }^{1,2 \& 4}$ Department of Entomology, Hajee Mohammad Danesh Science and Technology University (HMDSTU), Dinajpur. ${ }^{3}$ Department of Entomology, Bangladesh Agricultural University (BAU), Mymensingh, Bangladesh. 
et al., 1975; Bhalla et al., 2008). The adult beetles do not cause damage to the pulse grains by feeding but they mate and oviposite on grains and contaminate by excreta. The larva is solely responsible for the grain damage. The larvae destroy seeds by feeding inside partially or completely and make them unfit for human consumption (Atwal and Dhaliwal, 2005). About 4 to $98 \%$ loss of pulse seeds may be observed due to the infestation by the pulse beetle in storage (Mookherjee et al., 1970).

At present, pest control measures mostly rely on synthetic insecticides and fumigants in Bangladesh. But chemical protection measures may be resulted many serious drawbacks (Lee et al., 2001). Their extensive and indiscriminate use causes ecological imbalance, resistance of pesticides to pest, pest resurgence and outbreak of secondary pests, creates phytotoxicity, insecticidal residues in foods and feed (Mahmud et al., 2002; Ashamo, 2004; Nas, 2004). Moreover, continuous uses of insecticides leads to hazardous effect on pollinators, natural enemies e.g., predators, parasitoids and also caused the environmental pollution (Saxena, 1992; Nagarare and More, 1998; Hossain, 2001). Due to these hazards, globally scientists are trying to adopt alternative methods of pest control. The use of locally available indigenous plant materials in the control of pests are an ancient technology and used in many parts of the world (Roy et al., 2005). Various products of plants have been tried recently by researchers with a high degree of success as grain protectants against pulse beetle to reduce infestation in storage (Umrao and Verma, 2002; Epidi et al., 2008; Mahadi and Rahman, 2008). Keeping these views in mind, the present study was conducted to investigate the insecticidal effectiveness of some indigenous botanicals powders as grain protectant against $C$. chinensis on chickpea seeds.

\section{Materials and Method}

Collection and preparation of plant materials: The study was conducted in the Department of Entomology, Bangladesh Agricultural University (BAU), Mymensingh. Fresh leaves (17 indigenous plants) and seeds (4 plants) (Table 1) were collected from BAU campus and kept in the laboratory for 7 days for air drying followed by one day sun drying before making powder. After drying, the leaves and seeds were made powder separately by an electric grinder in the laboratory and passed through a 60 -mesh sieve to get fine powder. Later, each powder was kept separately in air tight plastic pot and stored at room temperature for experimental use.

Collection of chickpea seeds: Healthy chickpea (Cicer arietinum L.) seeds was purchased from the local market of Mymensingh town. The seeds were thoroughly cleaned, sun dried, cooled, and stored with $10 \pm 2 \%$ moisture content. The seeds were kept in air tight plastic container $(25 \mathrm{~cm}$ height $\mathrm{x} 15 \mathrm{~cm}$ dia.) at preserved at room temperature for study. 
Table 1. List of indigenous plant leaf and seed powders tested against $C$. chinensis.

\begin{tabular}{c|l|l|l|l}
\hline $\begin{array}{c}\text { Serial } \\
\text { no. }\end{array}$ & Common name & \multicolumn{1}{c}{ Scientific name } & \multicolumn{1}{c}{ Family } & $\begin{array}{c}\text { Plant part } \\
\text { used }\end{array}$ \\
\hline 1 & Akanda & Calotropis gigantia & Asclepiadaceae & Leaf \\
2 & Khoksa & Ficus bengalensis & Moraceae & Leaf \\
3 & Ghagra & Xanthium italicum & Compositae & Leaf \\
4 & Nayantara & Nerium indicum & Apocynaceae & Leaf \\
5 & Karabi & Nerium olender & Apocynaceae & Leaf \\
6 & Bel & Aegle marmelos & Rutaceae & Leaf \\
7 & Tobacco & Nicotiana tabacum & Solanaceae & Leaf \\
8 & Alamanda & Alamanda cathertica & Apocynaceae & Leaf \\
9 & Dondokalash & Leucas aspera & Labiateae & Leaf \\
10 & Marigold & Tagetes erecta & Compositae & Leaf \\
11 & Dhutura & Datura stromonium & Solanaceae & Leaf \\
12 & Chrysanthemum & Chrysanthemum segetum & Compositae & Leaf \\
13 & Karanja & Pongamia pinnata & Fabaceae & Leaf \\
14 & Neem & Azadirachta indica & Meliaceae & Leaf and Seed \\
15 & Biskatali & Polygonum hydropiper & Polygonaceae & Leaf and Seed \\
16 & Ata & Annona reticulata & Annonaceae & Leaf and Seed \\
17 & Castor & Ricinus communis & Euphorbiaceae & Leaf and Seed \\
\hline & & & &
\end{tabular}

Stock culture of pulse beetle: Pulse beetle was reared in the laboratory at ambient room temperature $\left(30 \pm 3^{\circ} \mathrm{C}\right)$ in glass jars $(47 \mathrm{~cm}$ height $\times 4 \mathrm{~cm}$ dia. $)$. Approximately, 200 adults of the collected pulse beetles were released in each jar containing $500 \mathrm{~g}$ of chickpea seeds and the mouth was closed with a piece of nylon cloth. The beetles were allowed for free mating and oviposition for 7 days. Then the beetles were separated from the seeds by sieving and seeds along with eggs were kept in the container $(8 \mathrm{~cm} \mathrm{~h} . \times 5 \mathrm{~cm}$ dia.) for emergence of next generation. After emergence, the newly emerged adults were collected and again allowed for further mating and oviposition with new seeds in different containers to maintain a series of stock culture of the test insect.

Screening procedure of plant powders: Screening of botanicals as grain protectants against $C$. chinensis was carried out following the primary and secondary screening.

Protocol of primary screening: Fifty grams of healthy chickpea seeds were weighed by an electric balance, taken in plastic container $(300 \mathrm{ml})$ and mixed it properly with tested leaf and seed powders separately at $20.0 \mathrm{~g} / \mathrm{kg}(\mathrm{w} / \mathrm{w})$ seeds. Five pairs of newly emerged one day old adult beetles of $C$. chinensis obtained 
from the stock culture were sexed and released in each plastic container along with an untreated control. After release of insects, the mouth of the plastic containers was closed with its porous lid. No plant materials were used in control treatment. Each treatment was replicated thrice. All treated containers were kept at ambient room temperature $\left(30 \pm 3^{\circ} \mathrm{C}\right)$ in the laboratory for oviposition and development of $C$. chinensis. Dead and alive beetles were removed after 7 days from each container and control. The effectiveness of plant materials as protectant against $C$. chinensis was assessed. For the determination of oviposition, 100 seeds were collected randomly from each plastic container of each treatment and examined under magnifying glass $(10 \mathrm{x})$. The total number of eggs deposited and number of seeds along with eggs (i.e., egg bearing seeds) were counted. After each observation, the grains were returned to the respective containers for the further development. Adults were removed and recorded daily after emergence. Infested and healthy seeds were separated, cleaned, counted, and finally weighed after completion of adult emergence. Seed infestation and weight loss were computed by using the following formulae:

Infestation $(\%)=\frac{N_{b}}{T_{n}} \times 100($ Enbakhare and Law-Ogbomo, 2002)

Where, $\mathrm{N}_{b}=$ Number of bored seeds, $\mathrm{T}_{n}=$ Total number of seeds

Weight loss $(\%)=\frac{U N d-D N u}{U(N d+N u)} \times 100(\mathrm{Lal}, 1988)$

Where, $\mathrm{U}=$ Weight of undamaged seeds, $\mathrm{D}=$ Weight of damaged seeds, $\mathrm{Nu}=$ Number of undamaged seeds, $\mathrm{Nd}=$ Number of damaged seeds.

Protocol of secondary screening: From the primary screening, only tobacco leaf powder (TLP) was found very effective to protect chickpea seeds against $C$. chinensis at $20.0 \mathrm{~g} / \mathrm{kg}$. On the other hand, TLP inhibited completely the progeny adult emergence. Therefore, TLP was further tested at lower doses of 10.0, 5.0, 2.5 , and $1.25 \mathrm{~g} / \mathrm{kg}$ seeds. Each dose was replicated five times along with an untreated control. The screening protocol and observations were same as followed in the primary screening.

Test on ovicidal and larvicidal effectiveness: To investigate whether tobacco leaf powder possesses ovicidal and larvicidal properties, another study was conducted. For this, 100 chickpea seeds along with one day old eggs (Ovicidal study) and 1-2 days old larvae (Larvicidal study) containing one egg or larva per seed were placed in each container and mixed properly with tobacco leaf powder @ 20.0, 10.0, 5.0, 2.5, and $1.25 \mathrm{~g} / \mathrm{kg}$ seeds along with an untreated control. After proper mixing, the mouth of the containers were closed with cap and left it undisturbed in the laboratory until adult emergence. The number of adult beetles 
were counted and recorded daily from the first to the last emergence and removed from the containers. After completion of adult emergence, the inhibition was computed by using the following formula as stated by Shukla et al. (2007).

Inhibition $(\%)=\frac{\text { Control mean }- \text { Treatment mean }}{\text { Control mean }} \times 100$

Seed germination test: To study the effect of TLP on seed viability and germination of chickpea seeds treated at different dose levels was carried out for a period of 3 months along with untreated control following the procedure with a slight modification as described by Enbakhare \& Law-Ogbomo (2002). For this, 100 seeds were placed in each Petridish $(120 \times 20 \mathrm{~mm})$ containing water soaked blotting paper (Whatman no. 1, UK) at the bottom. The Petridishes were placed in the laboratory under ambient room temperature $\left(30 \pm 3^{\circ} \mathrm{C}\right)$. Germinated seeds were counted, recorded and worked out the percent germination after incubation.

Statistical analyses: The collected data were analyzed followed by Completely Randomized Design (CRD) and analysis of variance (ANOVA). Data were transformed before analysis. The treatment mean values were compared by Duncan's New Multiple Range Test (DMRT).

\section{Results}

Primary screening: The number of eggs, egg bearing seeds, adult emergence, seed infestation and weight after infestation by $C$. chinensis on the chickpea seeds in different treatments differed significantly $(\mathrm{P}<0.05)$ with applied at 20 $\mathrm{g} / \mathrm{kg}$ seeds (Table 2). Among all the treatments, the highest number of eggs and egg bearing seeds per 100 seeds were found in the untreated control (97.33 and 77.67) while the lowest (16.33 and 15.0) was with tobacco leaf powder (TLP). Similarly, the highest number of adult emergence (198.33), seed infestation $(64.33 \%)$ and weight loss $(4.05 \%)$ were found in the untreated control. On the contrary, no adult emergence, seed infestation and weight loss were found when seeds were treated with TLP at $20 \mathrm{~g} / \mathrm{kg}$ seeds.

Secondary screening: From the primary screening, it was found that the TLP showed the best performance among all the tested powders against $C$. chinensis. Hence, it was further assessed at its lower doses in terms of ovicidal and larvicidal effectivity.

Effect of TLP on oviposition: The number of eggs of $C$. chinensis and egg bearing seeds on chickpea seeds treated with TLP differed significantly $(\mathrm{P}<0.05)$ among all the treatments (Table 3 ). The highest number of eggs was found in the untreated control (93.60), which was statistically similar to that at $1.25 \mathrm{~g} / \mathrm{kg}$ TLP treated seeds (84.40). But the lowest number of eggs (24.60) was recorded at $10.0 \mathrm{~g} / \mathrm{kg}$ seeds. The lowest number of egg bearing seeds per 100 seeds was recorded from TLP treated at $10.0 \mathrm{~g} / \mathrm{kg}$ seeds (23.40) while the highest in the untreated control (79.60). 
Table 2. Effects of indigenous plant powders on oviposition, adult emergence, seed infestation, and weight loss caused by $C$. chinensis on treated chickpea seeds.

\begin{tabular}{|c|c|c|c|c|c|}
\hline Treatments & $\begin{array}{l}\text { No. of } \\
\text { eggs/100 } \\
\text { seeds }\end{array}$ & $\begin{array}{c}\text { No. of egg } \\
\text { bearing } \\
\text { seeds/100 seed }\end{array}$ & $\begin{array}{l}\text { No. of adult } \\
\text { emergence }\end{array}$ & $\begin{array}{c}\text { Seed } \\
\text { infestation } \\
(\%)\end{array}$ & $\begin{array}{c}\text { Seed } \\
\text { wt loss } \\
(\%)\end{array}$ \\
\hline Akanda leaf & $\begin{array}{c}58.00 \mathrm{bc} \\
(1.76)\end{array}$ & $\begin{array}{c}47.00 \mathrm{c}-\mathrm{e} \\
(1.67)\end{array}$ & $\begin{array}{c}84.00 \mathrm{c}-\mathrm{e} \\
(1.92)\end{array}$ & $\begin{array}{c}27.56 \mathrm{~d}-\mathrm{f} \\
(31.62)\end{array}$ & $\begin{array}{l}1.65 \mathrm{fg} \\
(1.28)\end{array}$ \\
\hline Khoksa leaf & $\begin{array}{c}64.00 \mathrm{~b} \\
(1.81)\end{array}$ & $\begin{array}{l}52.00 \mathrm{bc} \\
(1.71)\end{array}$ & $\begin{array}{c}128.00 \mathrm{a}-\mathrm{c} \\
(2.11)\end{array}$ & $\begin{array}{l}40.34 \mathrm{~b} \\
(39.11)\end{array}$ & $\begin{array}{c}2.60 \mathrm{bc} \\
(1.61)\end{array}$ \\
\hline Ghagra leaf & $\begin{array}{c}75.33 \mathrm{ab} \\
(1.88)\end{array}$ & $\begin{array}{c}63.67 \mathrm{ab} \\
(1.80)\end{array}$ & $\begin{array}{c}122.67 \mathrm{a}-\mathrm{c} \\
(2.09)\end{array}$ & $\begin{array}{l}39.84 \mathrm{~b} \\
(39.12)\end{array}$ & $\begin{array}{c}2.34 \mathrm{~cd} \\
(1.53)\end{array}$ \\
\hline Nayantara leaf & $\begin{array}{l}29.00 \mathrm{f} \\
(1.46)\end{array}$ & $\begin{array}{c}23.67 \mathrm{~lm} \\
(1.37)\end{array}$ & $\begin{array}{l}74.33 \mathrm{c}-\mathrm{e} \\
(1.87)\end{array}$ & $\begin{array}{l}23.86 \mathrm{f} \\
(29.21)\end{array}$ & $\begin{array}{l}1.43 \mathrm{gh} \\
(1.20)\end{array}$ \\
\hline Neem leaf & $\begin{array}{c}22.67 \mathrm{~g} \\
(1.35)\end{array}$ & $\begin{array}{c}20.33 \mathrm{~m} \\
(1.31)\end{array}$ & $\begin{array}{l}32.67 \mathrm{f} \\
(1.52)\end{array}$ & $\begin{array}{l}11.84 \mathrm{~h} \\
(19.65)\end{array}$ & $\begin{array}{l}0.70 \mathrm{jk} \\
(0.83)\end{array}$ \\
\hline Karabi leaf & $\begin{array}{c}61.33 \mathrm{~b} \\
(1.79)\end{array}$ & $\begin{array}{l}47.33 \mathrm{~cd} \\
(1.67)\end{array}$ & $\begin{array}{l}91.67 \mathrm{~d}-\mathrm{e} \\
\quad(1.79)\end{array}$ & $\begin{array}{l}39.45 \mathrm{~b} \\
(38.89)\end{array}$ & $\begin{array}{c}2.36 \mathrm{bd} \\
(1.54)\end{array}$ \\
\hline Biskatali leaf & $\begin{array}{l}36.00 \text { ef } \\
(1.55)\end{array}$ & $\begin{array}{l}31.00 \mathrm{~h}-\mathrm{k} \\
(1.49)\end{array}$ & $\begin{array}{c}93.67 \mathrm{~b}-\mathrm{e} \\
(1.97)\end{array}$ & $\begin{array}{c}29.53 \\
(32.89)\end{array}$ & $\begin{array}{c}1.76 \mathrm{eg} \\
(1.32)\end{array}$ \\
\hline Bel leaf & $\begin{array}{c}46.33 \mathrm{c}-\mathrm{e} \\
(1.66)\end{array}$ & $\begin{array}{l}37.67 \mathrm{e}-\mathrm{h} \\
\quad(1.57)\end{array}$ & $\begin{array}{c}116.67 \mathrm{a}-\mathrm{c} \\
(2.07)\end{array}$ & $\begin{array}{c}32.81 \\
(34.92)\end{array}$ & $\begin{array}{c}2.04 \\
(1.42)\end{array}$ \\
\hline Ata leaf & $\begin{array}{c}60.67 \mathrm{~b} \\
(1.78)\end{array}$ & $\begin{array}{l}48.33 \mathrm{~cd} \\
(1.68)\end{array}$ & $\begin{array}{l}96.67 \mathrm{~b}-\mathrm{e} \\
(1.98)\end{array}$ & $\begin{array}{c}29.56 \mathrm{c}-\mathrm{e} \\
(32.91)\end{array}$ & $\begin{array}{c}1.69 \mathrm{e}-\mathrm{g} \\
(1.30)\end{array}$ \\
\hline Castor leaf & $\begin{array}{l}45.00 \mathrm{c}-\mathrm{e} \\
\quad(1.65)\end{array}$ & $\begin{array}{l}34.67 \mathrm{~g}-\mathrm{j} \\
\quad(1.54)\end{array}$ & $\begin{array}{l}91.0 \mathrm{~b}-\mathrm{e} \\
(1.96)\end{array}$ & $\begin{array}{l}27.19 \mathrm{i} \\
(31.41)\end{array}$ & $\begin{array}{l}1.60 \mathrm{fg} \\
(1.27)\end{array}$ \\
\hline Tobacco leaf & $\begin{array}{c}16.33 \mathrm{~h} \\
(1.21)\end{array}$ & $\begin{array}{c}15.00 \mathrm{n} \\
(1.17)\end{array}$ & $\begin{array}{l}0.00 \mathrm{~g} \\
(0.00)\end{array}$ & $\begin{array}{l}0.00 \mathrm{i} \\
(0.00)\end{array}$ & $\begin{array}{l}0.00 \mathrm{k} \\
(0.71)\end{array}$ \\
\hline Alamanda leaf & $\begin{array}{c}42.33 \mathrm{de} \\
(1.62)\end{array}$ & $\begin{array}{c}36.67 \mathrm{f}-\mathrm{i} \\
(1.56)\end{array}$ & $\begin{array}{c}107.0 \mathrm{~b}-\mathrm{d} \\
(2.03)\end{array}$ & $\begin{array}{c}31.17 \mathrm{~cd} \\
(33.91)\end{array}$ & $\begin{array}{l}1.86 \mathrm{ef} \\
(1.36)\end{array}$ \\
\hline Dondokalash leaf & $\begin{array}{c}65.67 \mathrm{~b} \\
(1.82)\end{array}$ & $\begin{array}{c}49.00 \mathrm{~cd} \\
(1.69)\end{array}$ & $\begin{array}{c}153.67 \mathrm{ab} \\
(2.19)\end{array}$ & $\begin{array}{l}43.09 \mathrm{~b} \\
(41.01)\end{array}$ & $\begin{array}{l}2.76 \mathrm{~b} \\
(1.66)\end{array}$ \\
\hline Marigold leaf & $\begin{array}{c}44.67 \mathrm{ce} \\
(1.65)\end{array}$ & $\begin{array}{c}34.67 \mathrm{~g}-\mathrm{j} \\
(1.54)\end{array}$ & $\begin{array}{c}96.33 \mathrm{~b}-\mathrm{e} \\
(1.98)\end{array}$ & $\begin{array}{c}26.93 \mathrm{~d}-\mathrm{f} \\
(31.24)\end{array}$ & $\begin{array}{l}1.68 \mathrm{fg} \\
(1.29)\end{array}$ \\
\hline Dhutura leaf & $\begin{array}{c}35.67 \mathrm{ef} \\
(1.55)\end{array}$ & $\begin{array}{c}30.33 \mathrm{~h}-\mathrm{k} \\
(1.48)\end{array}$ & $\begin{array}{c}85.67 \mathrm{c}-\mathrm{e} \\
(1.93)\end{array}$ & $\begin{array}{c}25.82 \mathrm{ef} \\
(30.49)\end{array}$ & $\begin{array}{l}1.58 \mathrm{fg} \\
(1.25)\end{array}$ \\
\hline Chrysanthemum leaf & $\begin{array}{l}30.67 \mathrm{f} \\
(1.48)\end{array}$ & $\begin{array}{c}26.67 \mathrm{kl} \\
(1.42)\end{array}$ & $\begin{array}{c}60.33 \mathrm{e} \\
(1.78)\end{array}$ & $\begin{array}{l}17.96 \mathrm{~g} \\
(25.05)\end{array}$ & $\begin{array}{l}1.06 \mathrm{i} \\
(1.03)\end{array}$ \\
\hline Karanja leaf & $\begin{array}{c}47.33 \mathrm{~cd} \\
(1.67)\end{array}$ & $\begin{array}{c}40.67 \mathrm{~d}-\mathrm{g} \\
\quad(1.61)\end{array}$ & $\begin{array}{c}128.00 \mathrm{a}-\mathrm{c} \\
(2.11)\end{array}$ & $\begin{array}{l}39.16 \mathrm{~b} \\
(38.71)\end{array}$ & $\begin{array}{l}2.38 \mathrm{~b} \\
(1.54)\end{array}$ \\
\hline Neem seed & $\begin{array}{c}20.33 \mathrm{gh} \\
(1.30)\end{array}$ & $\begin{array}{c}19.33 \mathrm{~m} \\
(1.28)\end{array}$ & $\begin{array}{c}30.67 \mathrm{f} \\
(1.48)\end{array}$ & $\begin{array}{l}11.33 \mathrm{~h} \\
(20.10)\end{array}$ & $\begin{array}{c}0.64 \mathrm{jk} \\
(0.80)\end{array}$ \\
\hline Ata seed & $\begin{array}{c}58.00 \mathrm{bc} \\
(1.76)\end{array}$ & $\begin{array}{l}44.67 \mathrm{c}-\mathrm{f} \\
\quad(1.65)\end{array}$ & $\begin{array}{l}92.00 \mathrm{~b}-\mathrm{e} \\
(1.96)\end{array}$ & $\begin{array}{c}28.51 \mathrm{c}-\mathrm{e} \\
(32.25)\end{array}$ & $\begin{array}{c}1.55 \mathrm{f}-\mathrm{h} \\
(1.24)\end{array}$ \\
\hline Castor seed & $\begin{array}{c}35.67 \mathrm{f} \\
(1.55)\end{array}$ & $\begin{array}{c}29.33 \mathrm{i}-1 \\
(1.46)\end{array}$ & $\begin{array}{c}82.33 \mathrm{c}-\mathrm{e} \\
(1.91)\end{array}$ & $\begin{array}{c}25.44 \text { ef } \\
(30.26)\end{array}$ & $\begin{array}{l}1.29 \mathrm{hi} \\
(1.13)\end{array}$ \\
\hline Biskatali seed & $\begin{array}{l}31.33 \\
(1.50)\end{array}$ & $\begin{array}{c}28.00 \mathrm{j}-1 \\
(1.45)\end{array}$ & $\begin{array}{c}87.67 \mathrm{~b}-\mathrm{e} \\
(1.91)\end{array}$ & $\begin{array}{c}28.90 \mathrm{c}-\mathrm{e} \\
(32.50)\end{array}$ & $\begin{array}{l}1.60 \mathrm{fg} \\
(1.26)\end{array}$ \\
\hline Control & $\begin{array}{c}97.33 \mathrm{a} \\
(1.98)\end{array}$ & $\begin{array}{c}77.67 \mathrm{a} \\
(1.88)\end{array}$ & $\begin{array}{c}198.33 \mathrm{a} \\
(2.30)\end{array}$ & $\begin{array}{l}64.33 \mathrm{a} \\
(53.33)\end{array}$ & $\begin{array}{l}4.05 \mathrm{a} \\
(2.01)\end{array}$ \\
\hline
\end{tabular}

Means in a column having the same letters are not significantly different at $5 \%$ by DMRT. Figures in the parenthesis are log transformed values. 
Effect of TLP on adult emergence: The number of adult emergence differed significantly $(\mathrm{P}<0.05)$ among the treatments $($ Table 3$)$. The highest number of adults were recorded in untreated control (194.60), whereas the lowest (23.20) in TLP treated seeds applied at $10.0 \mathrm{~g} / \mathrm{kg}$ seeds. The number of adult emergence was recorded 40.80, 71.0 and 154.20 when seeds were treated with TLP at 5.0, 2.5 and $1.25 \mathrm{~g} / \mathrm{kg}$ seeds, respectively.

Table 3. Effects of tobacco leaf powder on oviposition, adult emergence, seed infestation, and weight loss caused by $C$. chinensis including germination of treated chickpea seeds.

\begin{tabular}{|c|c|c|c|c|c|c|}
\hline $\begin{array}{l}\text { Dose } \\
\text { (g/kg } \\
\text { seed) }\end{array}$ & $\begin{array}{l}\text { No. of } \\
\text { eggs/100 } \\
\text { seeds }\end{array}$ & $\begin{array}{l}\text { No. of egg } \\
\text { bearing } \\
\text { seeds/ } 100 \\
\text { seeds }\end{array}$ & $\begin{array}{l}\text { No. of } \\
\text { adults } \\
\text { emerged }\end{array}$ & $\begin{array}{c}\text { Seed } \\
\text { infestation } \\
(\%)\end{array}$ & $\begin{array}{c}\text { Seed } \\
\text { wt loss } \\
(\%)\end{array}$ & $\begin{array}{c}\text { Seed } \\
\text { germination } \\
(\%)\end{array}$ \\
\hline 1.25 & $\begin{array}{c}84.40 \mathrm{a} \\
(1.92)\end{array}$ & $\begin{array}{c}67.20 \mathrm{~b} \\
(1.83)\end{array}$ & $\begin{array}{c}154.20 \mathrm{~b} \\
(2.19)\end{array}$ & $\begin{array}{l}42.45 \mathrm{~b} \\
(40.63)\end{array}$ & $\begin{array}{l}2.58 \mathrm{~b} \\
(1.61)\end{array}$ & $\begin{array}{l}91.00 \\
(9.54)\end{array}$ \\
\hline 2.5 & $\begin{array}{c}63.00 \mathrm{~b} \\
(1.80)\end{array}$ & $\begin{array}{c}55.60 \mathrm{c} \\
(1.75)\end{array}$ & $\begin{array}{c}71.00 \mathrm{c} \\
(1.85)\end{array}$ & $\begin{array}{l}24.58 \mathrm{c} \\
(29.70)\end{array}$ & $\begin{array}{l}1.52 \mathrm{c} \\
(1.23)\end{array}$ & $\begin{array}{l}90.40 \\
(9.51)\end{array}$ \\
\hline 5.0 & $\begin{array}{c}51.60 \mathrm{c} \\
(1.71)\end{array}$ & $\begin{array}{c}41.20 \mathrm{~d} \\
(1.61)\end{array}$ & $\begin{array}{c}40.80 \mathrm{~d} \\
(1.61)\end{array}$ & $\begin{array}{l}11.46 \mathrm{~d} \\
(19.77)\end{array}$ & $\begin{array}{l}0.70 \mathrm{~d} \\
(0.84)\end{array}$ & $\begin{array}{l}89.80 \\
(9.44)\end{array}$ \\
\hline 10.0 & $\begin{array}{c}24.60 \mathrm{~d} \\
(1.38)\end{array}$ & $\begin{array}{c}23.40 \mathrm{e} \\
(1.36)\end{array}$ & $\begin{array}{c}23.20 \mathrm{e} \\
(1.36)\end{array}$ & $\begin{array}{l}8.28 \mathrm{e} \\
(16.66)\end{array}$ & $\begin{array}{l}0.50 \mathrm{~d} \\
(0.71)\end{array}$ & $\begin{array}{l}89.60 \\
(9.46)\end{array}$ \\
\hline Control & $\begin{array}{c}93.60 \mathrm{a} \\
(1.97)\end{array}$ & $\begin{array}{c}79.60 \mathrm{a} \\
(1.90)\end{array}$ & $\begin{array}{c}194.60 \mathrm{a} \\
(2.29)\end{array}$ & $\begin{array}{l}63.55 \mathrm{a} \\
(52.86)\end{array}$ & $\begin{array}{l}4.21 \mathrm{a} \\
(2.05)\end{array}$ & $\begin{array}{l}91.40 \\
(9.56)\end{array}$ \\
\hline $\mathrm{CV}(\%)$ & 3.56 & 3.19 & 2.91 & 4.55 & 4.56 & NS \\
\hline
\end{tabular}

Means in a column having the same letter are not significantly different at $5 \%$ level by DMRT. Figures in the parenthesis are transformed values. NS = Not significant.

Effect of TLP on seed infestation and weight loss: It was observed from the data that the percentage of seed infestation and weight loss differed significantly $(\mathrm{P}<0.05)$ among the treatments (Table 3). The highest seed infestation was observed in untreated control $(63.55 \%)$ and the lowest $(8.28 \%)$ in TLP treated at $10.0 \mathrm{~g} / \mathrm{kg}$ seeds. Similarly, the highest seed weight loss was recorded in untreated control $(4.21 \%)$ and the lowest $(0.50 \%)$ in TLP applied at $10.0 \mathrm{~g} / \mathrm{kg}$ seed which was statistically similar to that applied at $5.0 \mathrm{~g} / \mathrm{kg}$ TLP treated seeds $(0.70 \%)$.

Effect of TLP on seed germination: The germination percentage of chickpea seeds treated with TLP did not differ significantly among the treatments (Table 3). The germination in different treatments including control ranged from 89.60 to $91.40 \%$ with no significant difference among them. 
Table 4. Number of adults of $C$. chinensis emerged from 100 eggs and larvae bearing chickpea seeds after treatment with tobacco leaf powder.

\begin{tabular}{|c|c|c|c|c|}
\hline \multirow{2}{*}{$\begin{array}{c}\text { Dose } \\
\text { (g/kg seed) }\end{array}$} & \multicolumn{2}{|c|}{ Egg bearing seed } & \multicolumn{2}{|c|}{ Larvae bearing seed } \\
\hline & $\begin{array}{c}\text { No. of adults } \\
\text { emerged }\end{array}$ & $\begin{array}{c}\text { Inhibition } \\
(\%)\end{array}$ & $\begin{array}{c}\text { No. of adults } \\
\text { emerged }\end{array}$ & $\begin{array}{c}\text { Inhibition } \\
(\%)\end{array}$ \\
\hline 1.25 & $\begin{array}{c}84.80 \mathrm{a} \\
(1.94)\end{array}$ & 6.61 & $\begin{array}{c}88.00 \mathrm{ab} \\
(1.94)\end{array}$ & 3.93 \\
\hline 2.5 & $\begin{array}{c}65.00 \mathrm{~b} \\
(1.81)\end{array}$ & 28.41 & $\begin{array}{c}79.00 \mathrm{~b} \\
(1.90)\end{array}$ & 13.76 \\
\hline 5.0 & $\begin{array}{c}38.00 \mathrm{c} \\
(1.58)\end{array}$ & 58.15 & $\begin{array}{c}64.00 \mathrm{c} \\
(1.80)\end{array}$ & 30.13 \\
\hline 10.0 & $\begin{array}{c}17.80 \mathrm{~d} \\
(1.24)\end{array}$ & 80.40 & $\begin{array}{c}55.50 \mathrm{~d} \\
(1.74)\end{array}$ & 39.41 \\
\hline 20.0 & $\begin{array}{l}0.00 \mathrm{e} \\
(0.00)\end{array}$ & 100.0 & $\begin{array}{c}37.20 \mathrm{e} \\
(1.57)\end{array}$ & 59.39 \\
\hline Control & $\begin{array}{c}90.80 \mathrm{a} \\
(1.96)\end{array}$ & - & $\begin{array}{c}91.60 \mathrm{a} \\
(1.96)\end{array}$ & - \\
\hline $\mathrm{CV}(\%)$ & 3.71 & - & 2.46 & - \\
\hline
\end{tabular}

Means in a column having the same letters are not significantly different at $5 \%$ level by DMRT. Figures in the parenthesis are log transformed values.

\section{Discussion}

The results of the present study revealed that among all the tested plant materials, tobacco leaf powder (TLP) had promising effects on the inhibition of oviposition and decreasing effect on adult emergence, seed infestation, and weight loss caused by $C$. chinensis. The TLP offered complete protection of chickpea seeds by applying the highest dose of $20.0 \mathrm{~g} / \mathrm{kg}$ seeds although few eggs (16.33) were deposited but failed to develop as subsequent adults (Table 2). While TLP at lower doses exerted its effectiveness in dose dependant manner. The lowest seed infestation and weight loss were found $8.28 \%$ and $0.50 \%$, respectively, when seeds were treated with TLP at $10.0 \mathrm{~g} / \mathrm{kg}$ seeds. The grain protectant properties of TLP might be attributed to its antiovipositional, ovicidal, and larvicidal properties. The results of the present findings are in close proximity with those of Govindan and Nelson (2008). They treated pulse seeds with ten botanicals and found that tobacco leaf powder (Nicotiana tabacum) along with Lictifers isora attributed the lowest number of eggs against the Callosobruchus maculatus. The present study showed that complete inhibition of adult emergence was found when egg bearing chickpea seeds were exposed to TLP treated at $20.0 \mathrm{~g} / \mathrm{kg}$ seed, but $59.39 \%$ inhibition was occurred when larvae bearing chickpea seeds were treated with the same dose of TLP (Table 4). These may be due to the mortality 
of eggs and/or larvae, or reduction of egg hatching. Bamaiyai et al. (2007) also opined that the ovicidal properties of botanical powders suppressed the emergence of pulse beetle. Chickpea seeds mixed with TLP have no adverse side effect on seed germination (Table 3) up to 3 months of storage. The present results are comparable to those of Paneru and Shivakoti (2001). The biological activity of TLP can be credited to its alkaloid contents, such as nicotine, nornicotine, neonicotine, anabasine, nicotyrine, metanicotine, etc. having deterrent action to insect (Prakash and Rao, 1996). Moreover, nicotine, a colourless liquid acts as fumigant and able to penetrate directly through insect integument. It also acts as non-persistent contact insecticide against various pests (Cremlyn, 1978). The semiochemical nature and pungent smell of TLP might alter the behaviour adversely and physiology of beetles markedly and thus preventing from oviposition. Moreover, fine powder of TLP could block the spiracles of the beetles, thereby, impairing respiration and leading to death.

Among other tested botanicals, neem leaf and neem seed powder significantly reduced the pulse beetle infestation and weight loss of chickpea seeds but failed to prevent to damage completely. None of the other tested powders applied at $20.0 \mathrm{~g} / \mathrm{kg}$ showed significant inhibition of adult emergence, reduction of seed infestation and weight loss of chickpea seeds. Varied effectiveness of different tested botanical powders indicated that the pest suppressing properties are not uniformly distributed among the tested plant powders. Significant level of success in the suppression of Callosobruchus sp. was reported by various authors with leaf powder of different botanicals including neem leaf and seed powders (Singh, 2003; Gundannavar and Deshpande, 2006; Sharma and Rathore, 2006; Lakshmi and Venugopal, 2007). However, with the finding of the present study, it may be opined that for ecofriendly management of $C$. chinensis, the TLP may be considered at farmer's level as it is cheaper, easily available, processable, and usable.

\section{References}

Ahmed, K. S., T. Itino and T. Ichikawa. 2003. Duration of developmental stages of Callosobruchus chinensis L. (Coleoptera: Bruchidae) on azuki bean and the effects of neem and sesame oils at different stages of their development. Pakistan J. Biol. Sci. 6 (10): 932-935.

Ashamo, M. O. 2004. Effects of some plant powders on yam moth, Dasyses rugosella Stainton (Lepidoptera: Tineidae). Biol. Sci. Res. Comm. 16: 41-46.

Aslam, M. 2004. Pest status of stored chickpea beetle, Callosobrachus chinensis L. on chickpea. J. Entomol. 1 (1): 28-33.

Atwal, A. S. and G. S. Dhaliwal. 2005. Agricultural Pests of South Asia and their Management. $5^{\text {th }}$ edition, Kalyani Publishers, New Delhi, India, 505P. 
Bamaiyai, L .J., I. S. Ndams, W. A. Toro and S. Odekina. 2007. Laboratory evaluation of mehogany (Khaya senegalensis Desv.) seed oil and seed powder for the control of Callosobruchus maculatus F. (Coleoptera: Bruchidae) on stored cowpea. J. Entomol. 4 (3): 237-242.

BBS (Bangladesh Bureau of Statistics). 2008. Statistical Year Book of Bangladesh. Bangladesh Bureau of Statistics, Planning Division, Ministry of Planning, Govt. of the People's Republic of Bangladesh, Dhaka, Bangladesh. Pp. 125-126.

Bhalla, S., K. Gupta, B. Lal, M. L. Kapur and R. K. Khetrapal. 2008. Efficacy of various non-chemical methods against pulse beetle, Callosobrachus maculatus (F.). ENDURE International Conference, Diversifying Crop Protection, France. Pp. 1-4.

Cremlyn, R. 1978. Pesticides: Preparation and Mode of Action, John Wiley and Sons, New York, Pp. 50-130.

Enbakhare, D.A. and K.E. Law-Ogbomo. 2002. Reduction of post harvest loss caused by Sitophilus zeamais (Motsch) in three varieties of maize treated with plant products. Post Harvest Sci. 1: 1-6.

Epidi, T. T., C.D. Nawanji. and S. Udoh. 2008. Efficacy of some plant species for the control of cowpea weevil (Callosobruchus maculatus) and maize weevil (Sitophilus zeamais). Int. J. Agri. Biol. 10: 588-590.

Govindan, K. and S. J. Nelson. 2008. Effect of mixtures of plant powder against pulse beetle, Callosobruchus maculatus (F.) (Coleoptera: Bruchidae). J. Plant Protect. Environ. 5 (1): 52-57.

Gundannavar, K. P. and V. K. Deshpande. 2006. Effect of indigenous products on seed quality and incidence of pulse beetle, Callosobruchus chinensis in different varieties of soybean. Karnataka J. Agric. Sci. 19 (2): 393-395.

Hossain, M. 2001. Samonnito Kitpatango Bebosthapona. In Bengali, Bangla Academy, Dhaka. $155 \mathrm{p}$.

Lakshmi, L.G. and M.S. Venugopal. 2007. Effectiveness of powdered plant products as grain protectants against the pulse beetle, Callosobruchus maculatus (F.). J. Entomol. Res. 31 (1): 75-78.

Lal, S. 1988. Estimation of losses and economics of specific storage losses. Regional Workshop on On-farm Storage Facilities and Design, Haripur, India, Pp. 79-89.

Lee, B., W. Choi, S. Lee and B. Park. 2001. Fumigant toxicity of essential oils and their constituent compounds towards the rice weevil, Sitophilus oryzae. Crop Prot. 20: 317-320.

Mahadi, S. H. A. and K. Rahman. 2008. Insecticidal effect of some species on Callosobruchus maculatus F. in blackgram seeds. Univ. J. Zool. 27: 47-50.

Mahmud M. K., M. M. H. Khan, M. Hussain, M. I. Alam and M. S. I. Afrad. 2002. Toxic effects of different plant oils on pulse beetle, Callosobruchus chinensis L. (Coleoptera: Bruchidae). J. Asiat. Soc. Bangladesh. 28 (1): 11-18. 
Mookherjee, P. B., M. G. Jotwani. T. D. Yadav and D. Sircar. 1970. Studies on the incidence and extent of damage due to insect pests in stored seeds. Leguminous and Vegetable seeds. Indian J. Entomol. 32 (4): 350-355.

Nagarare V.S. and G. D. More. 1998. Economics using bioagents against Helicoverpa armigera in pigeonpea. Indian J. Entomol. 60 (2): 203-206.

Nas, M. N. 2004. In vitro studies on some natural beverages as botanical pesticides against Erwinia amylovora and Curobacterium flaceumfaciensis subsp. Poinsettiae. Turk. J. Agric. 28: 57-61.

Paneru, R. B. and G. P. Shivakoti. 2001. Use of botanicals for the management of pulse beetle (Callosobruchus maculatus F.) in lentil. Nepal Agric. Res. J. 4 \& 5: 27-30.

Prakash, A. and Rao, J. 1996. Botanical Pesticides in Agriculture, CRC Press, New Delhi, India, 480P.

Rahman R., Huda S. and A.Hussain. 1975. Effect of gamma radiation on different stages of Callosobruchus maculatus F. Bangladesh J. Zool. 3: 103-109.

Roy B., M. R. Amin, M. N. Uddin, A. T. M. S. Islam, M. J. Islam and B. C. Halder. 2005. Leaf extracts of shiyalmutra (Blumera lacera) as botanical pesticides against lesser grain borer and rice weevil. J. Biol. Sci. 5 (2): 201-204.

Saxena S. C. 1992. Biology of Insects. Published by Raju Primlanifor Oxford and IBH Publishing Co. Pvt. Ltd., 66 Janapath, New Delhi 110001. P. 366.

Sharma, S. S. 1984. Review of literature of the losses caused by Callosobruchus chinensis (L.) (Coleoptera: Bruchidae) during storage of pulses. Bull. Grain Technol. 22 (1): 62-68.

Sharma, V. and Y. S. Rathore. 2006. Effect of some botanicals on adult's preference, oviposition and development of Callosobruchus chinensis (L.) on pigeon pea grains. Indian J. Entomol. 68 (1): 74-77.

Shukla, R., B. Srivastava, R. Kumar and N. K. Dubey. 2007. Potential of some botanical powders in reducing infestation of chickpea by Callosobruchus chinensis (L.) (Coleoptera: Bruchidae). J. Agric. Tech. 3 (1): 11-19.

Singh, S.C. 2003. Effect of neem leaf powder on infestation of the pulse beetle, Callosobruchus chinensis in stored khesari. Indian J. Entomol. 65 (2): 188-192.

Umrao, R. S. and R. A Verma. 2002. Effectiveness of some plant products against pulse beetle on pea. Indian J. Entomol. 64 (4): 451-453. 